\title{
Secoisolariciresinol diglucoside prevents the oxidative stress-induced apoptosis of myocardial cells through activation of the JAK2/STAT3 signaling pathway
}

\author{
GUIQIONG HUANG ${ }^{1 *}$, XIAOFANG HUANG ${ }^{1 *}$, MIN LIU $^{2 *}$, YUE HUA $^{1 *}$, BO DENG $^{3}$, WEN JIN $^{4}$, \\ WEN YAN $^{4}$, ZHANGBIN TAN $^{1}$, YIFEN WU $^{1,5}$, BIN LIU $^{1,2,6}$ and YINGCHUN ZHOU ${ }^{1}$ \\ ${ }^{1}$ School of Traditional Chinese Medicine; ${ }^{2}$ School of Public Health, \\ Southern Medical University, Guangzhou, Guangdong 510515; ${ }^{3}$ Department of Cardiology, \\ The Second Affiliated Hospital of Guangzhou Medical University, Guangzhou, Guangdong 510260; \\ ${ }^{4}$ Guangdong Second Provincial General Hospital, Guangzhou, Guangdong 510317; ${ }^{5}$ Department of Oncology, \\ Dongguan People's Hospital, Dongguan, Guangdong 523059; ${ }^{6}$ Center for Cancer and Inflammation Research, \\ School of Chinese Medicine, Hong Kong Baptist University, Hong Kong, SAR 999077, P.R. China
}

Received June 28, 2017; Accepted January 10, 2018

DOI: $10.3892 / \mathrm{ijmm} .2018 .3560$

\begin{abstract}
Myocardial cell apoptosis mediated by oxidative stress has previously been identified as a key process in ischemic heart disease. Secoisolariciresinol diglucoside (SDG), a polyphenolic plant lignan primarily found in flaxseed, has been demonstrated to effectively protect myocardial cells from apoptosis. In the present study, the role of the Janus kinase 2 (JAK2)/signal transducer and activator of transcription 3 (STAT3) was investigated in mediating the protective effect of SDG. Findings of the present study revealed that treatment with $\mathrm{H}_{2} \mathrm{O}_{2}$ reduced cell viability and induced apoptosis in $\mathrm{H} 9 \mathrm{C} 2$ rat cardiomyocytes. However, SDG was able to reduce the effect of $\mathrm{H}_{2} \mathrm{O}_{2}$ in a dose-dependent manner. $\mathrm{H}_{2} \mathrm{O}_{2}$ reduced the expression level of phosphorylated STAT3 and inhibited the levels of B-cell lymphoma-extra-large and induced myeloid leukemia cell differentiation protein, which are the STAT3 target genes. Conversely, SDG rescued phosphorylation of STAT3 and increased the levels of STAT3 target genes. Treatment with SDG alone led to a dose-dependent increased phosphorylation of JAK2 and STAT3, without activating Src. Furthermore, the anti-apoptotic effects of SDG were partially abolished by a JAK2/STAT3 inhibitor.
\end{abstract}

Correspondence to: Professor Bin Liu or Professor Yingchun Zhou, School of Traditional Chinese Medicine, Southern Medical University, 1838 Guangzhou Dadaobei Road, Guangzhou, Guangdong 510515, P.R. China

E-mail:xmhoolv@163.com

E-mail: zhychun@126.com

*Contributed equally

Key words: secoisolariciresinol diglucoside, apoptosis, Janus kinase 2, signal transducer and activator of transcription 3, cardiomyocyte, oxidative stress
In addition, molecular docking revealed that SDG may bind to the protein kinase domain of JAK2, at a binding energy of $-8.258 \mathrm{kcal} / \mathrm{mol}$. Molecular dynamics simulations revealed that JAK2-SDG binding was stable. In conclusion, activation of the JAK2/STAT3 signaling pathway contributed to the anti-apoptotic activity of SDG, which may be a potential JAK2 activator.

\section{Introduction}

Ischemic heart disease (IHD) is among the leading causes of disease-associated mortality worldwide (1). Cardiac oxidative injury during IHD, including myocardial infarction (MI) and myocardial ischemia/reperfusion (I/R), leads to cardiomyocyte apoptosis and results in physiopathological processes, such as fibrosis, hypertrophy and ventricular remodeling, ultimately leading to heart failure (2). Excessive oxidative stress may inhibit pro-survival signaling, reduce cell viability and trigger cell apoptosis (3). Activating pro-survival signaling pathways and rescuing cardiomyocytes from apoptosis has been demonstrated to be an effective IHD treatment strategy (4).

Flaxseed (Linum usitatissimum L.) is a functional food and has potential health benefits in a variety of diseases, including MI, atherosclerosis, diabetes and hyperlipidaemia (5-7). Secoisolariciresinol diglucoside (SDG) is a plant lignan extracted from flaxseed that belongs to the polyphenolic class of chemical compounds. It has been previously demonstrated that SDG is a potential agent for cardioprotection (8). SDG has been demonstrated previously to reduce infarct size and to recover aortic flow and function in an I/R model $(8,9)$. It was also determined that SDG improved left ventricular function, inhibited ventricular remodeling, and promoted angiogenesis in an MI model via upregulation of vascular endothelial growth factor protein expression levels (9). In addition, a previous study demonstrated that SDG prevented oxidative stress-induced inflammation and apoptosis in cultured cardiomyocytes (10). These findings suggested that SDG is a 
potential agent for cardioprotection. However, the underlying mechanisms remain to be elucidated.

Janus kinase 2 (JAK2)/signal transducer and activator of transcription 3 (STAT3) has been demonstrated to be a key signaling pathway in cardioprotection $(11,12)$. Activation of JAK2 and STAT3 is enhanced by ischemic or hypoxia preconditioning in I/R or hypoxia/reoxygenation cell models $(13,14)$. Previous studies revealed that suppression of JAK 2 by the JAK inhibitor AG490 eliminated the cardioprotective effects of ischemic preconditioning in myocardial I/R $(13,14)$. In addition, deficiency of STAT3 has been demonstrated to increase apoptosis in an inflammation-induced heart damage and myocardial I/R model $(15,16)$. However, whether SDG may protect cardiomyocytes against oxidative stress-induced apoptosis through the JAK2/STAT3 signaling pathway remains to be determined. The present study used an $\mathrm{H}_{2} \mathrm{O}_{2}$-induced in vitro cardiomyocyte apoptosis model to investigate whether the JAK2/STAT3 pathway was involved in the anti-apoptotic effect of SDG.

\section{Materials and methods}

Cells and reagents. The $\mathrm{H} 9 \mathrm{C} 2$ cell line, which derives from embryonic rat heart cells, was purchased from the Cell Bank of Type Culture Collection of Chinese Academy of Sciences (Shanghai, China). SDG was purchased from Sigma-Aldrich (Merck Millipore; Darmstadt, Germany; purity $>97 \%$ as determined by HPLC). JAK2/STAT3 inhibitor WP1066 was purchased from Santa Cruz Biotechnology, Inc. (Dallas, TX, USA; purity $>97 \%$ as determined by HPLC). Antibodies against poly-(ADP-ribose) polymerase (PARP, cat. no. 9532), p-JAK2 (Tyr-1007/Tyr-1008, cat. no. 3771), JAK2 (cat. no. 3230), p-STAT3 (Tyr-705, cat. no. 9145), p-STAT3 (Ser-727, cat. no. 9134), STAT3 (cat. no. 12640), Src (cat. no. 2191), B-cell lymphoma-extra-large (Bcl-xL, cat. no. 2762) and induced myeloid leukemia cell differentiation protein (Mcl-1, cat. no. 94296) were purchased from Cell Signaling Technology, Inc. (Danvers, MA, USA). P-Src (Tyr-418, cat. no. ab40660) was purchased from Abcam (Cambridge, MA, USA). Annexin V/propidium iodide (PI) Apoptosis Detection kit was purchased from BD Biosciences (San Jose, CA, USA). Remaining reagents used in the study were obtained from commercial sources.

MTT cell viability assay. H9C2 cells were plated at a density of $5.0 \times 10^{3}$ cells per well in 96-well plates. Cells were treated with 50,100 and $150 \mu \mathrm{M}$ SDG with or without $5 \mu \mathrm{M}$ JAK2/STAT3 inhibitor for $24 \mathrm{~h}$ at $37^{\circ} \mathrm{C}$ and were treated with $400 \mu \mathrm{M} \mathrm{H}_{2} \mathrm{O}_{2}$ for $2 \mathrm{~h}$ at $37^{\circ} \mathrm{C}$. Cell viability was detected using an MTT assay as previously described (17). Briefly, following treatment, the culture medium was removed from each well and replaced with $0.5 \mathrm{mg} / \mathrm{ml} \mathrm{MTT}$ solution for $4 \mathrm{~h}$ at $37^{\circ} \mathrm{C}$. Following incubation, culture medium was removed and $150 \mu$ ldimethyl sulfoxide (Sigma-Aldrich; Merck Millipore) per well was added for formazan solubilization. The formazan was quantified by determining the absorbance at $490 \mathrm{~nm}$, using an microplate reader (Thermo Fisher Scientific, Inc., Walham, MA, USA). The viability of the cardiomyocytes $(\%)$ was calculated as follows: Viability (\% of control)=optical density (OD) mean test group/OD mean control group x100.
Measurement of lactate dehydrogenase ( $\mathrm{LDH}$ ) levels. H9C2 cells were plated at a density of $5.0 \times 10^{3}$ cells/well in 96-well plates. $\mathrm{H} 9 \mathrm{C} 2$ cells were pretreated with 50, 100 and $150 \mu \mathrm{M}$ SDG for $24 \mathrm{~h}$ at $37^{\circ} \mathrm{C}$, and were stimulated with $400 \mu \mathrm{M} \mathrm{H}_{2} \mathrm{O}_{2}$ for $2 \mathrm{~h}$ at $37^{\circ} \mathrm{C}$. Subsequently, 96-well plates were centrifuged at $400 \mathrm{x} \mathrm{g}$ for $5 \mathrm{~min}$ at $4^{\circ} \mathrm{C}$ to obtain the cellular supernatant and the LDH release was quantified using LDH Release assay kit (cat. no. C0016; Beyotime Institute of Biotechnology, Haimen, China) following the manufacturer's protocol.

Flow cytometric quantification of apoptosis. Flow cytometry (BD Biosciences) was performed to quanitfy cell apoptosis. H9C2 cells were treated with indicated doses of SDG with/without the JAK2/STAT3 inhibitor for $24 \mathrm{~h}$ and then treated with $\mathrm{H}_{2} \mathrm{O}_{2}$ for $2 \mathrm{~h}$. The apoptosis index of $\mathrm{H} 9 \mathrm{C} 2$ cells was detected using the Annexin V/PI Apoptosis Detection kit following the manufacturer's protocol. The data was analyzed using FlowJo version 6.0 (Tree Star, Inc., Ashland, OR, USA).

Western blot assay. Western blot assay was performed as previously described (18). Briefly, protein extracts of cultured cells were prepared in RIPA lysis buffer (Beyotime Institute of Biotechnology, Haimen, China). Protein concentration of each sample was detected using BCA protein assay kit (Thermo Fisher Scientific, Inc.). Protein samples $(15 \mu \mathrm{g})$ were separated by $10 \%$ sodium dodecyl sulfate gel electrophoresis, and were transferred onto polyvinylidene fluoride membranes (Merck Millipore), subsequently blocked in Tris-buffered saline including $5 \%$ non-fat milk. Membranes were incubated at $4^{\circ} \mathrm{C}$ overnight with the corresponding primary antibodies [PARP 1:1,000, p-STAT3 (Tyr-705) 1:1,000, p-STAT3 (Ser-727), STAT3 1:1,000, p-JAK2 (Tyr-1007/ Tyr-1008) 1:1,000, JAK2 1:1,000, p-Src (Tyr-418) 1:1,000, Src 1:1,000, Mcl-1 1:1,000, Bcl-xL 1:1,000, GAPDH 1:5,000]. Then membranes were incubated with horseradish peroxidase-conjugated anti-rabbit secondary antibody (cat no. 7074; 1:1,000; Cell Signaling Technology, Inc.) for $1 \mathrm{~h}$ at $37^{\circ} \mathrm{C}$. Each experiment was repeated three times. Band intensities were quantified using ImageJ version 1.47 (National Institutes of Helath, Bethesda, MD, USA) and normalized to the intensities of GAPDH loading control bands.

Molecular simulation study. Molecular docking and dynamics analyses (MD) were performed to investigate the mechanism of interaction between SDG and JAK2. X-ray crystallographic structures of JAK2 (PDB ID: 4C61) (19) were used as starting structures for prediction of the binding site on SDG (CID: 9917980). The crystallographic structures of the JAK2 protein were prepared for molecular docking by removing water and co-crystallized ligands. Energy minimization of ligands was performed using YASARA version 16.9.23 (www.yasara.org). The protein kinase domain of JAK2 was defined in the present study as the protein-ligand binding site of JAK2. Molecular docking simulation was performed using Autodock Vina version 1.1.2 (vina.scripps.edu; The Scripps Research Institute, La Jolla, CA, USA) (20). PyMol version 1.3 and Ligplot ${ }^{+}$version 1.4 were used to visualize the JAK2-SDG complex $(21,22)$. The conformation with the lowest binding energy (lower is better) was used as star conformation for MD simulation. 

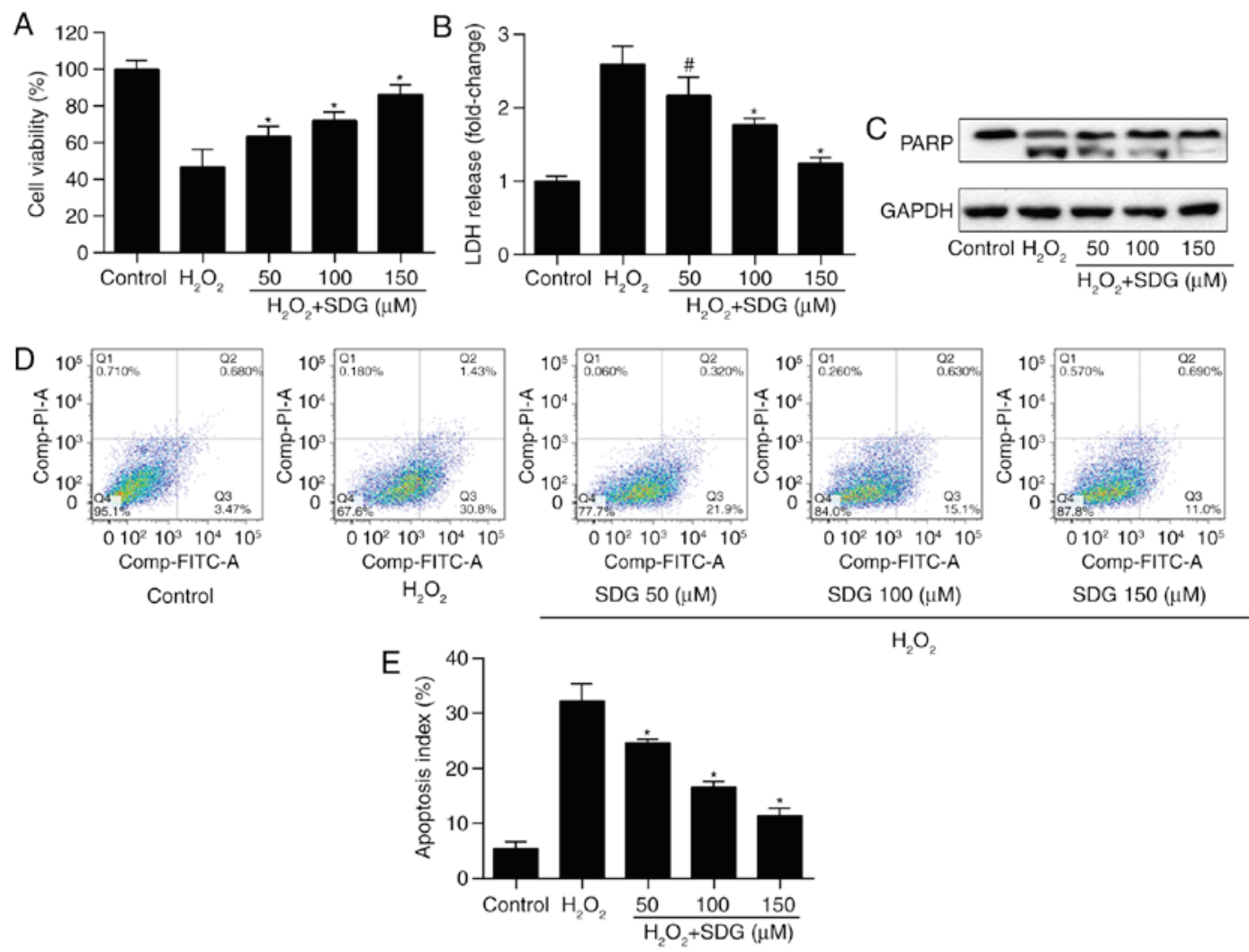

Figure 1. Pretreatment with SDG reduced oxidative stress-induced apoptosis of $\mathrm{H} 9 \mathrm{C} 2$ cells. $\mathrm{H} 9 \mathrm{C} 2$ cells were pretreated with indicated doses of SDG (50, 100 and $150 \mu \mathrm{M}$ ) for $24 \mathrm{~h}$, and then stimulated with $\mathrm{H}_{2} \mathrm{O}_{2}(400 \mu \mathrm{M})$ for $2 \mathrm{~h}$ and (A) cell viability $(\mathrm{n}=6)$, (B) $\mathrm{LDH}$ release were measured ( $\left.\mathrm{n}=3\right)$. (C) PARP expression levels were detected. (D) Representative Annexin V/PI stainings and (E) quantification of the apoptotic index in $\mathrm{H} 9 \mathrm{C} 2$ cells $(\mathrm{n}=3$ ). Data are presented as the mean \pm standard deviation. ${ }^{\prime \prime} \mathrm{P}<0.05,{ }^{*} \mathrm{P}<0.01$ vs. the $\mathrm{H}_{2} \mathrm{O}_{2}$ group. $\mathrm{LDH}$, lactate dehydrogenase; PARP, poly(ADP-ribose) polymerase; SDG, secoisolariciresinol diglucoside.

MD simulation was performed using YASARA (23). All simulations were run with the AMBER ff99sb force field option selected. The JAK2-SDG complex was solvated in a cube box of $0.9 \% \mathrm{NaCl}$, and the distance between solute and the box was $5 \AA$ A. Simulations were run at $298 \mathrm{~K}$ followed by simulated annealing minimizations which start at $298 \mathrm{~K}$ and velocities were scaled down with 0.9 every ten steps for 500 steps. Subsequently, 70 ns MD simulation was performed with a time step of $2 \mathrm{fsec}$ and the simulation snapshots were saved every 100 psec.

Statistical analysis. Data are presented as the mean \pm standard deviation. Calculations were performed using SPSS version 13.0 (SPSS, Inc., Chicago, IL, USA). One-way analysis of variance was performed to determine the significance of multiple comparisons. Least Significant Difference was used for statistical comparisons between treatment groups. $\mathrm{P}<0.05$ was considered to indicate a statistically significant difference.

\section{Results}

Pretreatment with SDG attenuates oxidative stress-mediated apoptosis in $\mathrm{H} 9 \mathrm{C} 2$ cells. To investigate whether SDG prevents $\mathrm{H} 9 \mathrm{C} 2$ from oxidative stress-mediated apoptosis, cells were pretreated with various doses of SDG for $24 \mathrm{~h}$ and subsequently stimulated with $\mathrm{H}_{2} \mathrm{O}_{2}$ for $2 \mathrm{~h}$. Cell viability of $\mathrm{H} 9 \mathrm{C} 2$ was reduced by $\mathrm{H}_{2} \mathrm{O}_{2}$ treatment, but was increased in a dose-dependent manner in SDG treatment groups (Fig. 1A). LDH release assay indicated that treatment with $\mathrm{H}_{2} \mathrm{O}_{2}$ significantly increased LDH release, whereas SDG treatment reduced it, in a dose-dependent manner (Fig. 1B). Subsequently, the protective effect of SDG against $\mathrm{H}_{2} \mathrm{O}_{2}$-induced apoptosis was examined, by detecting PARP expression and performing Annexin/PI assay. It was determined that SDG treatment led to a dose-dependent reduction of cleaved PARP expression levels (Fig. 1C). Accordingly, SDG treatment significantly reduced the apoptosis index of H9C2 cells (Fig. 1D and E). This suggested that SDG protected cardiomyocytes against oxidative stress-mediated cell apoptosis.

$S D G$ pretreatment rescued STAT3 signaling activation in $\mathrm{H}_{2} \mathrm{O}_{2}$-treated $\mathrm{H} 9 \mathrm{C} 2$. It has been previously established that STAT3 activation prevents cardiomyocyte apoptosis $(15,16)$. Therefore, whether SDG increased the activity of STAT3 in $\mathrm{H}_{2} \mathrm{O}_{2}$-treated $\mathrm{H} 9 \mathrm{C} 2$ cells was investigated. It was demonstrated that SDG caused a dose-dependent increase in phosphorylated STAT3 (p-STAT3, at Tyr-705 and Ser-727) expression levels (Fig. 2A-C). It was further examined whether SDG affected STAT3-target genes in H9C2 cells. Mcl-1 and Bcl-xL are target genes of STAT3 involved in cell apoptosis (24). It was observed that Mcl-1 and Bcl-xL protein levels were significantly increased in $\mathrm{H}_{2} \mathrm{O}_{2}$-treated $\mathrm{H} 9 \mathrm{C} 2$ cells following SDG treatment (Fig. 2D-F). These findings indicated that SDG rescued STAT3 signaling activation in $\mathrm{H}_{2} \mathrm{O}_{2}$-treated $\mathrm{H} 9 \mathrm{C} 2$ cardiomyocytes. 

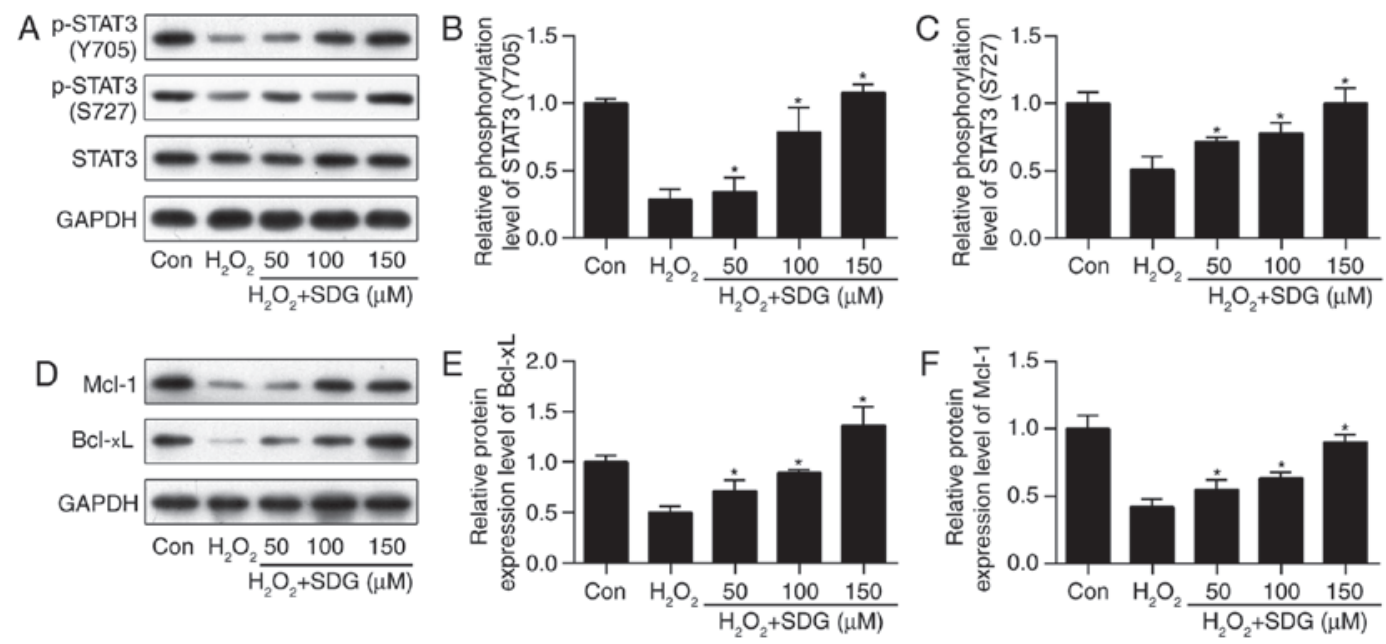

Figure 2. SDG treatment activated JAK2/STAT3 signaling in H9C2 cells. H9C2 cells were pretreated with indicated dose of SDG and subsequently stimulated with $\mathrm{H}_{2} \mathrm{O}_{2}$. Protein expression levels of (A) p-STAT3 and STAT3 and (B and C) quantification of their relative phosphorylation levels were determined by western blotting $(n=3)$. Western blot analysis of (D) Mcl-1 and Bcl-xL, and (E and F) their quantification ( $\mathrm{n}=3$ ). Data are presented as the mean \pm standard deviation. " $\mathrm{P}<0.01$ vs. the $\mathrm{H}_{2} \mathrm{O}_{2}$ group. Bcl-xL, B-cell lymphoma-extra-large; Mcl-1, induced myeloid leukemia cell differentiation protein; p, phosphorylated; PARP, poly(ADP-ribose) polymerase; SDG, secoisolariciresinol diglucoside; STAT3, signal transducer and activator of transcription 3.

Pretreatment with SDG alone enhances activation of STAT3 and JAK2, but not Src. To determine whether SDG activated the STAT3-associated signaling pathway directly or through inhibition of oxidative stress, phosphorylation levels of STAT3 following pretreatment with indicated concentrations of SDG for $24 \mathrm{~h}$ were investigated. As illustrated in Fig. 3A-C, pretreatment with SDG led to a dose-dependent increase of expression levels of p-STAT3 at the Tyr-705 and Ser-727 sites. It has been previously established that STAT3 may be activated by JAK 2 and non-receptor tyrosine kinase Src $(25,26)$. Therefore, the present study determined if activation of JAK2 and/or Src occurred in SDG-treated H9C2 cells. As presented in Fig. 3D-F, phosphorylation of Src did not change following SDG treatment, and expression of p-JAK2 was increased in SDG-treated groups. These findings suggested that SDG may directly activate the JAK2/STAT3 signaling pathway.

Inhibition of JAK2/STAT3 attenuates the anti-apoptotic effects of SDG. In order to determine if the anti-apoptotic effect of SDG in $\mathrm{H} 9 \mathrm{C} 2$ cells was due to the activation of the JAK2/STAT3 signaling pathway, WP1066 which is a JAK2/STAT3 inhibitor (27) was used. As illustrated in Fig. 4A-D, phosphorylation of JAK2 and STAT3 was increased by SDG treatment; however, it was reduced in $\mathrm{H} 9 \mathrm{C} 2$ cells treated with with WP1066 and with/without SDG. These results indicated that co-treatment with WP1066 inhibited the activation effect of SDG on the JAK2/STAT3 signaling pathway. Following treatment with SDG and/or WP1066 for $24 \mathrm{~h}, \mathrm{H} 9 \mathrm{C} 2$ cells were treated with $\mathrm{H}_{2} \mathrm{O}_{2}$ for $2 \mathrm{~h}$, and phosphorylation of JAK2 and STAT3 was measured by western blot analysis. As demonstrated in Fig. 4E-J, co-treatment of WP1066 significantly reduced the phosphorylation levels of JAK2 and STAT3, as well as the expression levels of Mcl-1 and Bcl-xL, were increased by SDG in $\mathrm{H}_{2} \mathrm{O}_{2}$-treated $\mathrm{H} 9 \mathrm{C} 2$ cells. These results confirmed that WP1066 abolished the activation effect of SDG on the JAK2/STAT3 signaling pathway. Finally, MTT and Annexin V/PI staining was performed to determine the role of JAK2/STAT3 signaling in the anti-apoptotic effect of SDG. As demonstrated in Fig. 4K, viablity of H9C2 cells was significantly reduced by treatment with $\mathrm{H}_{2} \mathrm{O}_{2}$ or WP1066 alone. SDG treatment rescued the viability of $\mathrm{H}_{2} \mathrm{O}_{2}$-stimulated H9C2 cells, whereas inhibition of JAK2/STAT3 impeded the protective effects of SDG on cell viability, reducing it from 82.5 to $50.3 \%$. Accordingly, inhibition of JAK2/STAT3 partly abolished the anti-apoptotic effects of SDG on apoptotic rate from 10.8 to $25.6 \%$ (Fig. 4L). These results implied that the anti-apoptotic effect of SDG was, partially at least, due to the activation of the JAK2/STAT3 signaling pathway.

SDG directly targets the kinase domain of JAK2. The present findings demonstrated that SDG significantly enhanced the phosphorylation levels of JAK2. Therefore, it is possible that SDG directly activates JAK2. Subsequently, the interaction between the protein kinase domain of JAK2 and SDG was investigated, by molecular docking and MD simulation. SDG molecule was docked into the protein kinase domain of the JAK2 protein (PDB: 4C61). The binding energy of the JAK2-SDG complex was $-8.258 \mathrm{kcal} / \mathrm{mol}$. Three- and two-dimensional structures of the JAK2-SDG complex are presented in Fig. 5A and B. Two hydrogen bonds were formed between SDG and JAK2 Leu-932 and Gln-853. The distance of hydrogen bonds between SDG and JAK2 Leu-932 and Gln-853 were $\AA, 2.85$ and $2.80 \AA$, respectively. Two hydrogen bonds were formed between SDG and Arg-938 of JAK2, and the distance of hydrogen bonds between them was 2.99 and $2.95 \AA$, respectively. The best conformation of JAK2-SDG was used as the star conformation for the MD simulation by YASARA. Fig. 5C demonstrates the evolution of backbone atoms and the root-mean-square deviation (RMSD) of the complex with respect to the minimized structure. The RMSD track in the last $50 \mathrm{nsec}$ fluctuated from 2.52 to $4.14 \AA$ in the unbound state of the JAK2 protein, and from 2.32 to $3.26 \AA$ in the JAK2-SDG complex. These results suggested strong binding between the kinase domain of JAK2 and SDG, which indicated that SDG may directly target JAK2. 

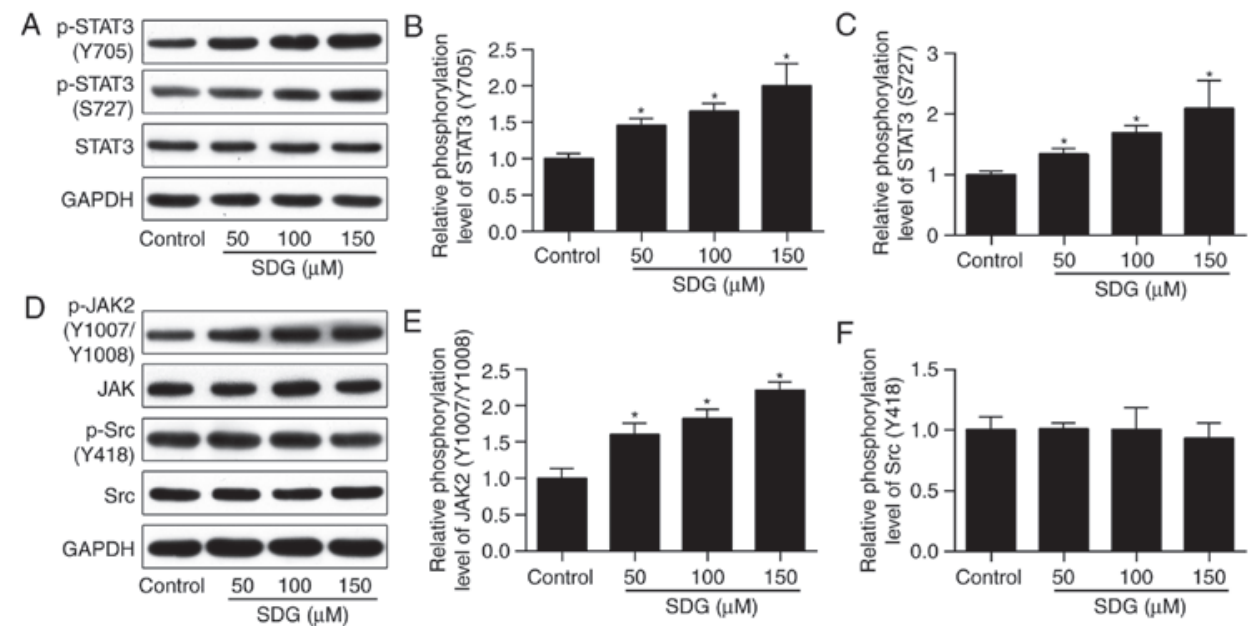

Figure 3. SDG treatment activated STAT3 and JAK2 but not Src. Following treatment with indicated doses of SDG, expression levels of (A) p-STAT3 (Tyr-705), p-STAT3 (Ser-727) and STAT3 were detected and (B and C) quantification of their relative phosphorylation levels was measured by western blotting $(n=3)$. Expression levels of (D) p-JAK2, JAK2, p-Src and Src were detected and (E and F) quantification of their relative phosphorylation levels were determine using western blot analysis $(\mathrm{n}=3)$. Data are presented as the mean \pm standard deviation. ${ }^{*} \mathrm{P}<0.01$ vs. the control group. JAK2, Janus kinase 2; $\mathrm{p}$, phosphorylated; SDG, secoisolariciresinol diglucoside; Src, sarcoma; STAT3, signal transducer and activator of transcription 3.
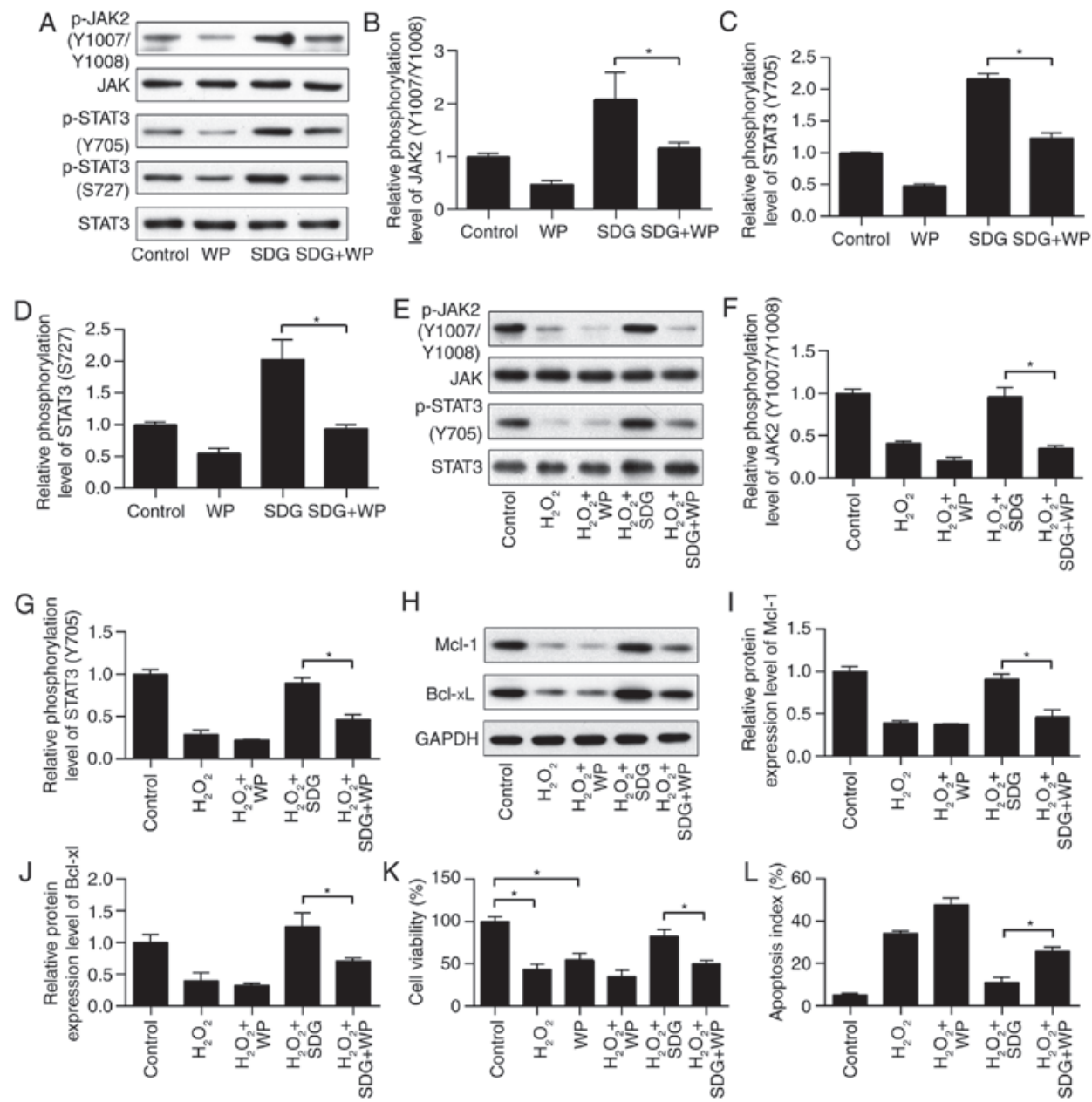

Figure 4. Inhibition of JAK2/STAT3 signaling attenuated the anti-apoptotic effects of SDG. H9C2 cells were pretreated with SDG $(150 \mu \mathrm{M})$ with or without the JAK2/STAT3 inhibitor WP1066 (5 $\mu \mathrm{M})$. Expression levels of (A) p-JAK2, JAK2, p-STAT3 (Tyr-705), STAT3 (Ser-727) and STAT3 and (B-D) quantification of their relative phosphorylation levels, as measured by western blot analysis $(\mathrm{n}=3)$. $\mathrm{H} 9 \mathrm{C} 2$ cells were pretreated with SDG $(150 \mu \mathrm{M})$ with or without the JAK2/STAT3 inhibitor WP1066 $(5 \mu \mathrm{M})$, following stimulation with $\mathrm{H}_{2} \mathrm{O}_{2}(400 \mu \mathrm{M})$ for $2 \mathrm{~h}$. Expression levels of (E) p-JAK2, JAK2, p-STAT3 (Tyr-705), and STAT3 and (F and G) quantification of their relative phosphorylation levels, as measured by western blot analysis $(\mathrm{n}=3)$. Expression levels of $(\mathrm{H}) \mathrm{Mcl}-1$ and Bcl-xL and (I and J) quantification of their relative protein levels, as measured by western blot analysis ( $\mathrm{n}=3$ ). (K) Viability of H9C2 cells ( $\mathrm{n}=6)$ and (L) apoptosis ratio $(n=3)$, as measured by MTT and Annexin V/PI staining, respectively. Data are presented as the mean \pm standard deviation. "P<0.01. Bcl-xL, B-cell lymphoma-extra-large; Mcl-1, induced myeloid leukemia cell differentiation protein; JAK2, Janus kinase 2; p, phosphorylated; SDG, secoisolariciresinol diglucoside; STAT3, signal transducer and activator of transcription 3; WP, WP1066 JAK2/STAT3 inhibitor. 


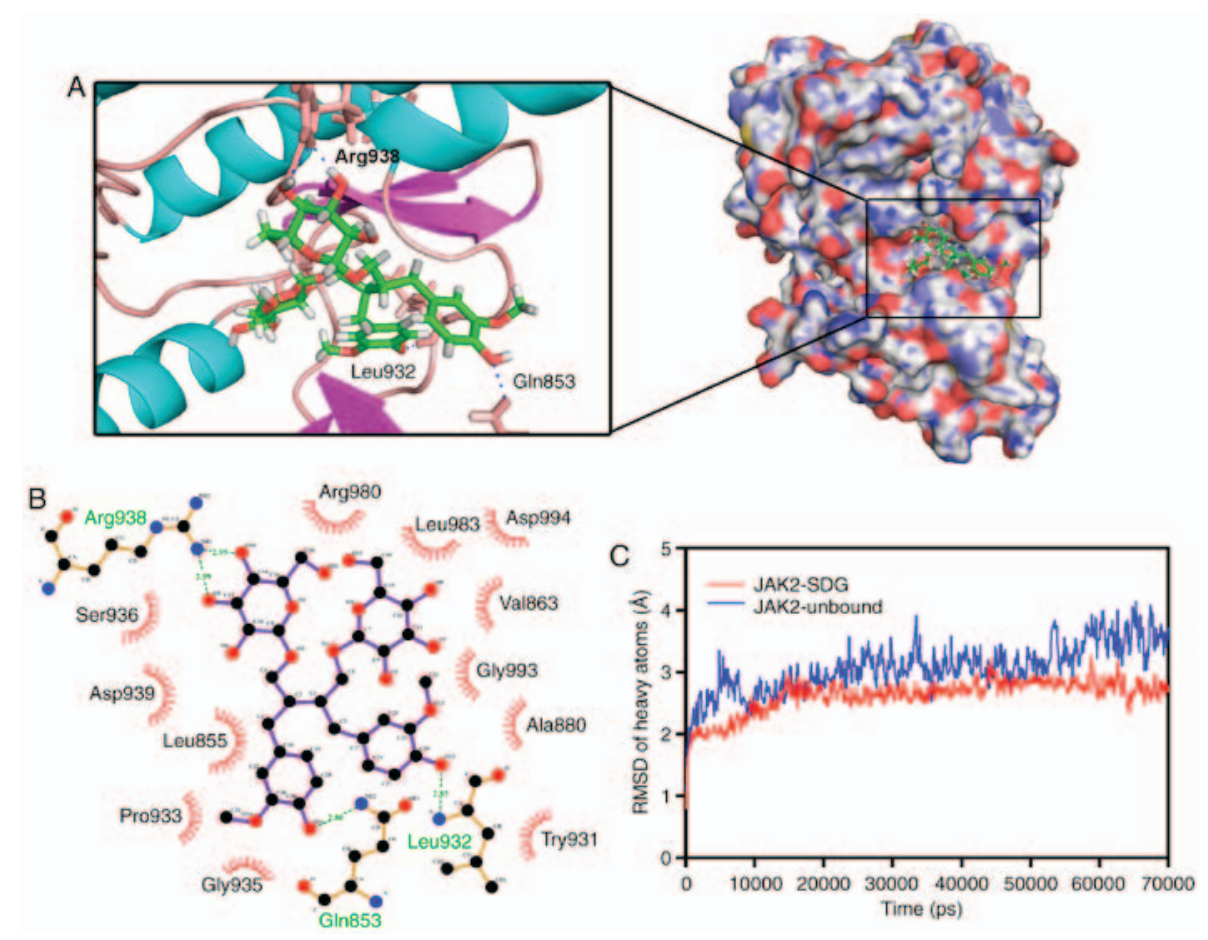

Figure 5. Molecular simulation depicting the interaction between SDG and JAK2. (A) Three-dimensional crystal structure of SDG in complex with JAK2 (PDB ID: 4C61). SDG is in green, and the hydrogen bond is indicated by a blue dotted line. (B) Two-dimensional map of SDG in complex with JAK2 the four hydrogen bonds formed between the two molecules are indicated in green. (C) Plots of RMSD of the backbone of JAK2 unbound (blue) and JAK2-SDG complex (red). Arg, arginine; Gln, glutamine; JAK2, Janus kinase 2; Leu, leucine; RMSD, root mean square deviation; SDG, secoisolariciresinol diglucoside.

\section{Discussion}

SDG is isolated from flaxseed and has a number of health benefits, such as cardioprotective activity (8). In the present study, the anti-apoptotic effect of SDG was investigated in $\mathrm{H}_{2} \mathrm{O}_{2}$-treated $\mathrm{H} 9 \mathrm{C} 2$ cells and it was demonstrated that SDG pretreatment significantly reduced apoptosis of cells in the presence of $\mathrm{H}_{2} \mathrm{O}_{2}$, which is in agreement with a previous study (10).

The STAT3 signaling pathway is important for cell resistance to apoptosis. Inhibition of STAT3 has been previously reported in heart failure in human patients and animal models $(28,29)$. The present study revealed that $\mathrm{H}_{2} \mathrm{O}_{2}$ significantly reduced the activation of STAT3. A previous study revealed that treatment with $\mathrm{H}_{2} \mathrm{O}_{2}$ increased STAT3 activation in $\mathrm{H} 9 \mathrm{C} 2$ cells (30); however, the duration of exposure to $\mathrm{H}_{2} \mathrm{O}_{2}(30 \mathrm{~min})$ was shorter than that in the present study. SDG pretreatment significantly rescued phosphorylation levels of STAT3 in $\mathrm{H}_{2} \mathrm{O}_{2}$-treated cardiomyocytes. Following phosphorylation at Tyr-705 and Ser-727, STAT3 translocates into the nucleus and binds to specific DNA response elements in the promoter region of STAT3-target genes (31). Expression levels of Bcl-xL and Mcl-1, which are STAT3-target genes, were reduced following $\mathrm{H}_{2} \mathrm{O}_{2}$ exposure, and increased in SDG pretreatment groups. These results indicated that treatment with SDG rescued the activation of the STAT3 pathway in $\mathrm{H}_{2} \mathrm{O}_{2}$-treated cardiomyocytes.

In the present study, $\mathrm{H}_{2} \mathrm{O}_{2}$ significantly reduced the activation of STAT3. Whether SDG directly activated the STAT3-associated signaling pathway or inhibited oxidative stress was also investigated. Cardiomyocytes were treated with SDG alone and the phosphorylation levels of STAT3 were quantified which demonstrated that SDG significantly activated
STAT3, indicating that SDG directly activated STAT3-related signaling pathway. However, the possibility that SDG inhibited oxidative stress level by activating oxidative stress-associated signaling pathway cannot be ruled out. It has been previously demonstrated that STAT3 may be activated by upstream tyrosine kinases including Src and JAK2 $(31,32)$. To determine whether SDG activated STAT3 or its upstream signaling molecules, phosphorylation levels of JAK2 and Src were investigated. In the present study, SDG increased JAK2 phosphorylation in a dose-dependent manner, whereas phosphorylation of Src was not altered. These results indicated that SDG may activate STAT3 signaling through activation of JAK2.

Subsequently, the function of JAK2/STAT3 in the anti-apoptotic action of SDG was investigated using WP1066, a JAK2 and STAT3 inhibitor (27). The anti-apoptotic effects of SDG were counteracted by WP1006, suggesting that JAK2/STAT3 is the pharmacological target of SDG and activation of JAK2/STAT3 by SDG may be a novel treatment strategy for cardiac protection. It has been previously demonstrated that SDG induced angiogenesis in human coronary arteriolar endothelial cells (8). It was also demonstrated that flaxseed extract, which contains SDG, induced proliferation and migration of human dermal fibroblasts and may be a novel therapy for infected wounds (33).

The present findings suggested that SDG may target JAK2. Therefore, molecular docking and MD simulation was performed to determine whether SDG may bind the protein kinase domain of JAK2. The binding energy of the JAK2-SDG complex indicated a strong binding ability. Furthermore, MD simulation results demonstrated that JAK2-SDG binding conformation was stable. These results indicated that SDG directly targets JAK2, acting as a potential JAK2 agonist. 
In conclusion, SDG was demonstrated to exert anti-apoptotic activities. These effects were partially mediated via activation of the JAK2/STAT3 signaling pathway and it was demonstrated that SDG may be a potential JAK2 agonist. As JAK2/STAT3 activation has an essential role in anti-apoptosis $(28,29)$, the present findings may provide a novel perspective for investigating the mechanism by which SDG or flaxseed may aid in treating IHD.

\section{Acknowledgements}

The present study was supported by the National Natural Science Foundation of China (grant nos. 81673805, 81373575, 81673949, and 81601779), Guangdong Natural Science Foundation (grant nos. 2014A030313495 and 2014A030310210), the Science and Technology Planning Project of Guangdong Province (grant nos. 2014A020221013 and 2014A020221059), the Science Technology and Innovation Committee of Shenzhen (grant no. JCYJ20150630164505508) and the Traditional Chinese Medicine Bureau of Guangdong Province (grant no. 20161260).

\section{Competing interests}

The authors declare that they have no competing interests.

\section{References}

1. Benjamin EJ, Blaha MJ, Chiuve SE, Cushman M, Das SR, Deo R, de Ferranti SD, Floyd J, Fornage M, Gillespie C, et al: Heart disease and stroke statistics-2017 update: A report from the American heart association. Circulation 135: e146-e603, 2017.

2. Singh SS and Kang PM: Mechanisms and inhibitors of apoptosis in cardiovascular diseases. Curr Pharm Des 17: 1783-1793, 2011.

3. Zhao ZQ: Oxidative stress-elicited myocardial apoptosis during reperfusion. Curr Opin Pharmacol 4: 159-165, 2004

4. Hamacher-Brady A, Brady NR and Gottlieb RA: The interplay between pro-death and pro-survival signaling pathways in myocardial ischemia/reperfusion injury: Apoptosis meets autophagy. Cardiovasc Drugs Ther 20: 445-462, 2006.

5. Slavova-Kazakova A, Karamać M, Kancheva V and Amarowicz R: Antioxidant activity of flaxseed extracts in lipid systems. Molecules 21: E17, 2015.

6. Prasad K and Dhar A: Flaxseed and diabetes. Curr Pharm Des 22: 141-144, 2016.

7. Prasad K: Flaxseed and cardiovascular health. J Cardiovasc Pharmacol 54: 369-377, 2009.

8. Penumathsa SV, Koneru S, Thirunavukkarasu M, Zhan L, Prasad K and Maulik N: Secoisolariciresinol diglucoside: Relevance to angiogenesis and cardioprotection against ischemia-reperfusion injury. J Pharmacol Exp Ther 320: 951-959, 2007.

9. Penumathsa SV, Koneru S, Zhan L, John S, Menon VP, Prasad K and Maulik N: Secoisolariciresinol diglucoside induces neovascularization-mediated cardioprotection against ischemia-reperfusion injury in hypercholesterolemic myocardium. J Mol Cell Cardiol 44: 170-179, 2008.

10. Puukila S, Bryan S, Laakso A, Abdel-Malak J, Gurney C, Agostino A, Belló-Klein A, Prasad K and Khaper N Secoisolariciresinol diglucoside abrogates oxidative stress-induced damage in cardiac iron overload condition. PLoS One 10: e122852, 2015.

11. Boengler K, Hilfiker-Kleiner D, Drexler H, Heusch G and Schulz R: The myocardial JAK/STAT pathway: From protection to failure. Pharmacol Ther 120: 172-185, 2008.

12. Bolli R, Stein AB, Guo Y, Wang OL, Rokosh G, Dawn B, Molkentin JD, Sanganalmath SK, Zhu Y and Xuan YT: A murine model of inducible, cardiac-specific deletion of STAT3: Its use to determine the role of STAT3 in the upregulation of cardioprotective proteins by ischemic preconditioning. J Mol Cell Cardiol 50: 589-597, 2011.
13. Xuan YT, Guo Y, Han H, Zhu Y and Bolli R: An essential role of the JAK-STAT pathway in ischemic preconditioning. Proc Natl Acad Sci USA 98: 9050-9055, 2001.

14. Suleman N, Somers S, Smith R, Opie LH and Lecour SC: Dual activation of STAT-3 and Akt is required during the trigger phase of ischaemic preconditioning. Cardiovasc Res 79: 127-133, 2008.

15. Hilfiker-Kleiner D, Hilfiker A, Fuchs M, Kaminski K, Schaefer A, Schieffer B, Hillmer A, Schmiedl A, Ding Z, Podewski E, et al: Signal transducer and activator of transcription 3 is required for myocardial capillary growth, control of interstitial matrix deposition, and heart protection from ischemic injury. Circ Res 95: 187-195, 2004.

16. Jacoby JJ,Kalinowski A, Liu MG, Zhang SS, Gao Q, Chai GX, Ji L, Iwamoto Y, Li E, Schneider M, et al: Cardiomyocyte-restricted knockout of STAT3 results in higher sensitivity to inflammation, cardiac fibrosis, and heart failure with advanced age. Proc Natl Acad Sci USA 100: 12929-12934, 2003.

17. Liu B, Zhang J, Liu W, Liu N, Fu X, Kwan H, Liu S, Liu B, Zhang S, Yu Z and Liu S: Calycosin inhibits oxidative stress-induced cardiomyocyte apoptosis via activating estrogen receptor- $\alpha / \beta$. Bioorg Med Chem Lett 26: 181-185, 2016.

18. Liu B, Liu NN, Liu WH, Zhang SW, Zhang JZ, Li AQ and Liu SM: Inhibition of lectin-like oxidized low-density lipoprotein receptor-1 reduces cardiac fibroblast proliferation by suppressing GATA binding protein 4. Biochem Biophys Res Commun 475: 329-334, 2016.

19. Su Q, Ioannidis S, Chuaqui C, Almeida L, Alimzhanov M, Bebernitz G, Bell K, Block M, Howard T, Huang S, et al: Discovery of 1-methyl-1H-imidazole derivatives as potent Jak2 inhibitors. J Med Chem 57: 144-158, 2014.

20. Trott $\mathrm{O}$ and Olson AJ: AutoDock Vina: Improving the speed and accuracy of docking with a new scoring function, efficient optimization, and multithreading. J Comput Chem 31: 455-461, 2010

21. Yang Y, Hu B and Lill MA: WATsite2.0 with PyMOL Plugin: Hydration site prediction and visualization. Methods Mol Biol 1611: 123-134, 2017.

22. Laskowski RA and Swindells MB: LigPlot ${ }^{+}$: Multiple ligand-protein interaction diagrams for drug discovery. J Chem Inf Model 51: 2778-2786, 2011

23. Krieger E, Koraimann G and Vriend G: Increasing the precision of comparative models with YASARA NOVA-a self-parameterizing force field. Proteins 47: 393-402, 2002.

24. Teng Y, Ross JL and Cowell JK: The involvement of JAK-STAT3 in cell motility, invasion, and metastasis. JAKSTAT 3: e28086, 2014.

25. Nam S, Xie J, Perkins A, Ma Y, Yang F, Wu J, Wang Y, Xu RZ, Huang W, Horne DA and Jove R: Novel synthetic derivatives of the natural product berbamine inhibit Jak2/Stat3 signaling and induce apoptosis of human melanoma cells. Mol Oncol 6: 484-493, 2012

26. Niu G, Bowman T, Huang M, Shivers S, Reintgen D, Daud A, Chang A, Kraker A, Jove R and Yu H: Roles of activated Src and Stat 3 signaling in melanoma tumor cell growth. Oncogene 21: 7001-7010, 2002 .

27. Song B, Jin H, Yu X, Zhang Z, Yu H, Ye J, Xu Y, Zhou T, Oudit GY, Ye JY, et al: Angiotensin-converting enzyme 2 attenuates oxidative stress and VSMC proliferation via the JAK2/STAT3/SOCS3 and profilin-1/MAPK signaling pathways. Regul Pept 185: 44-51, 2013.

28. Podewski EK, Hilfiker-Kleiner D, Hilfiker A, Morawietz H, Lichtenberg A, Wollert KC and Drexler H: Alterations in Janus kinase (JAK)-signal transducers and activators of transcription (STAT) signaling in patients with end-stage dilated cardiomyopathy. Circulation 107: 798-802, 2003.

29. Zhang W, Qu X, Chen B, Snyder M, Wang M, Li B, Tang Y, Chen H, Zhu W, Zhan L, et al: Critical roles of STAT3 in $\beta$-adrenergic functions in the heart. Circulation 133: 48-61, 2016.

30. Reed DK and Arany I: Sex hormones differentially modulate STAT3-dependent antioxidant responses during oxidative stress in renal proximal tubule cells. In Vivo 28: 1097-1100, 2014.

31. Carpenter RL and Lo HW: STAT3 target genes relevant to human cancers. Cancers (Basel) 6: 897-925, 2014.

32. Chen-Scarabelli C, Saravolatz IL, McCaukey R, Scarabelli G, Di Rezze J, Mohanty B, Barry S, Latchman D, Georgiadis V, McCormick J, et al: The cardioprotective effects of urocortin are mediated via activation of the Src tyrosine kinase-STAT3 pathway. JAKSTAT 2: e24812, 2013.

33. Czemplik M, Kulma A, Bazela K and Szopa J: The biomedical potential of genetically modified flax seeds overexpressing the glucosyltransferase gene. BMC Complement Altern Med 12: 251, 2012 . 